\title{
LA CONDUCTA ULTERIOR DE LAS PARTES COMO REGLA PRINCIPAL DE INTERPRETACIÓN DE LOS TRATADOS
}

\author{
Fabián NovAK*
}

\begin{abstract}
SUMARIO: 1 . ASPECTOS GENERALES.-2. LAS REGLAS DE INTERPRETACIÓN DE LOS TRATADOS. - 3. LA CONDUCTA ULTERIOR DE LAS PARTES COMO REGLA DE INTERPRETACIÓN PRINCIPAL.-3.1. Regla general obligatoria.-3.2. Regla convencional y consuetudinaria.-3.3. Regla aplicable en forma combinada.-3.4. Contenido y carácter de prueba objetiva.-3.5. El término «ulterior».-3.6. Alcances del acuerdo ulterior.-3.7. Alcances de la práctica ulterior.-3.8. Diferencias entre los acuerdos ulteriores y la práctica ulterior.-3.9. Las conferencias de Estados parte.-3.10. Los órganos de expertos.-3.11. Los órganos de control.-3.12. Alcances modificatorios de esta regla.-3.13. La conducta ulterior y la interpretación evolutiva.-3.14. Criterios de valoración de la regla.-4. REFLEXIÓN FINAL.
\end{abstract}

\section{ASPECTOS GENERALES}

La interpretación de los tratados es ante todo una operación intelectual, en virtud de la cual se busca establecer el contenido y alcance de sus disposiciones, de forma tal que se le dé un cabal y adecuado cumplimiento. En otros términos, la interpretación permite precisar o aclarar cualquier duda que pueda surgir de la lectura del instrumento internacional como también conocer cuál fue el propósito que las partes quisieron alcanzar al celebrarlo, lo que reviste una significación capital en el cumplimiento de una obligación convencional ${ }^{1}$.

La interpretación puede ser llevada a cabo por un sinfín de personas, sujetos e instituciones. Así, puede ser efectuada por los propios Estados parte de un tratado en conjunto (interpretación auténtica), en cuyo caso tendrá efectos jurídicos erga omnes; por un tribunal judicial o arbitral internacional,

\footnotetext{
* Profesor Principal de Derecho internacional público de la Facultad de Derecho de la Pontificia Universidad Católica del Perú (fnovak@pucp.edu.pe).

1 YASSEN, M., "L'interprétation des traités d'après la Convention de Vienne sur le droit des traités", Recueil des Courses de l'Académie de Droit International, vol. 151, 1976, t. III, pp. 1-114 ; JENNINGS, R. y WatTs, A., Oppenheim's International Law, vol. I: Peace Introduction and Part I, Londres, Longman, 1993, p. 1267.
} 
cuyos efectos serán solo atribuibles a las partes de la controversia y sobre la disputa en cuestión; por los Estados parte de un tratado de manera individual (interpretación unilateral) a través de sus distintos órganos (ejecutivo, legislativo, judicial, etc.) con efectos solo al interior de las fronteras del Esta$\mathrm{do}^{2}$; como también esta operación puede ser efectuada por la doctrina de los publicistas de manera individual o a través de las distintas asociaciones que periódicamente se encargan de sentar posición sobre los avances del Derecho internacional, en cuyo caso no existe efecto jurídico alguno, empero esta interpretación sí puede ejercer una influencia considerable en cómo deben ser interpretadas las disposiciones de un tratado.

En relación a cómo se debe interpretar un tratado, según Georges Berlia se distinguen tres métodos básicos de interpretación. El primero, el método textual, que incide en el análisis del texto, en el vocabulario empleado y en el sentido ordinario de los términos. El segundo, el método subjetivo, que busca establecer la voluntad de las partes en el texto y fuera de él, analizando para ello los trabajos preparatorios y la conducta ulterior de las partes. Finalmente, el tercero, es el método funcional o teleológico, que pretende determinar el fin o función para el que fue celebrado el tratado, buscando cumplir su objeto y fin ${ }^{3}$.

Cada uno de estos métodos ha dado lugar, a su vez, a la aparición de reglas específicas de interpretación, las mismas que han sido recogidas taxativamente en la Convención de Viena sobre Derecho de los Tratados de 1969" como veremos a continuación.

Antes de ello, debemos adelantar que el presente estudio estará dedicado a desarrollar tan solo una de estas reglas, cual es la de la conducta ulterior de las partes, y ello fundamentalmente por tres razones: la primera, es que se trata de una regla principal de interpretación de los tratados, que necesariamente debe ser tomada en cuenta en cualquier proceso de interpretación que se lleve a cabo; la segunda, es que esta regla ha tenido un especial desarrollo doctrinario y jurisprudencial en los últimos años; y la tercera, es que muy recientemente (agosto de 2018) la Comisión de Derecho Internacional (CDI) de la Organización de las Naciones Unidas (ONU) aprobó el Proyecto de conclusiones sobre los acuerdos ulteriores y la práctica ulterior en relación con la interpretación de los tratados ${ }^{5}$, luego de un largo y apreciable trabajo desarrollado por el relator Georg Nolte. Todo esto justifica detenernos un momento a señalar cuáles son las características y alcances que presenta esta importante regla de interpretación, en el Derecho internacional actual.

\footnotetext{
2 Ehrlich, L., "L'interprétation des traités», Recueil des Courses de l'Académie de Droit International, vol. 24, 1928, t. III, pp. 1-139, esp. pp. 35 y 38 .

3 Berlia, G., "Contribution à l'interprétation des traités», Recueil des Courses de l'Académie de Droit International, t. I, 1965, núm. 114, pp. 283-331, esp. p. 295.

${ }^{4}$ Convención de Viena sobre el Derecho de los Tratados de 23 de mayo de 1969. U.N. Doc A/ CONF.39/27 (1969), 1155 UNTS 331, entrada en vigor el 27 de enero de 1980.

5 Comisión de Derecho InTERnacional (CDI), Proyecto de conclusiones sobre los acuerdos ulteriores y la práctica ulterior en relación con la interpretación de los tratados, Informe Anual, $70 .^{\circ}$ periodo de sesiones (30 de abril a 1 de junio y 2 de julio a 10 de agosto de 2018), Suplemento núm. 10 (A/73/10).
} 


\section{LAS REGLAS DE INTERPRETACIÓN DE LOS TRATADOS}

Tal como se señaló en el punto anterior, la Convención de Viena sobre el Derecho de los Tratados de 1969 dispone en su art. 31 las reglas generales de interpretación. Así señala:

«Artículo 31

Regla general de interpretación

1. Un tratado deberá interpretarse de buena fe conforme al sentido corriente que haya de atribuirse a los términos del tratado en el contexto de estos y teniendo en cuenta su objeto y fin.

2. Para los efectos de la interpretación de un tratado, el contexto comprenderá, además del texto, incluidos su preámbulo y anexos:

a) Todo acuerdo que se refiera al tratado y haya sido concertado entre todas las partes con motivo de la celebración del tratado.

b) Todo instrumento formulado por una o más partes con motivo de la celebración del tratado y aceptado por las demás como instrumento referente al tratado.

3. Juntamente con el contexto, habrá de tenerse en cuenta:

a) Todo acuerdo ulterior entre las partes acerca de la interpretación del tratado o de la aplicación de sus disposiciones;

b) Toda práctica ulteriormente seguida en la aplicación del tratado por la cual conste el acuerdo de las partes acerca de la interpretación del tratado;

c) Toda norma pertinente de Derecho internacional aplicable en las relaciones entre las partes.

4. Se dará a un término un sentido especial si consta que tal fue la intención de las partes».

El art. 31 de la Convención —que se inspiró a su vez en una resolución del Institut de Droit International de $1956^{6}$, así como en los Principios formulados por Gerald Fitzmaurice ${ }^{7}$ — fue aprobado en 1969 por 97 votos a favor y ningún voto en contra, lo que da cuenta del alto nivel de consenso existente sobre las reglas que contiene. Fue por ello que estas mismas reglas fueron establecidas en el art. 31 de la Convención de Viena sobre el Derecho de los Tratados entre Estados y Organizaciones Internacionales y entre Organizaciones Internacionales de $1986^{8}$. Respecto de la aceptación general de estas reglas, señala Eduardo Jiménez de Aréchaga:

«Las normas jurídicas relativas a la interpretación de los Tratados constituyen una de las sanciones de la Convención de Viena que fueron adoptadas por la

\footnotetext{
6 Institut de Droit InTERnational, Annuaire, 1956, pp. 364-365.

7 Fitzmaurice, G., «The Law and Procedure of the International Court of Justice 1951-4: Treaty Interpretation and other Treaty Points», British Year Book of International Law, vol. XXXIII, 1957, pp. 203-293, esp. pp. 211-212.

8 Convención de Viena sobre Derecho de los Tratados entre Estados y Organizaciones Internacionales y entre Organizaciones Internacionales de 21 de marzo de 1986. Official Records of the United Nations Conference on the Law of Treaties between States and International Organizations or between International Organizations, vol. II (United Nations publication, Sales No. E.94.V.5).
} 
Conferencia sin voto disidente alguno y, por consiguiente, pueden ser consideradas como declaratorias del Derecho en vigor. Los cuatro artículos dedicados a la interpretación de los tratados están basados en la jurisprudencia establecida por la CIJ y destilan la esencia de ciertos principios fundamentales que pueden considerarse como reglas de Derecho internacional sobre el tema y no meramente directivas generales, aplicables a todos los tratados, cualquiera sea su naturaleza o contenido» ${ }^{9}$.

Como se puede observar, este artículo consagra un pluralismo de métodos y reglas de interpretación de los tratados; sin embargo, señala como punto de partida dos principios que deben guiar todo proceso interpretativo: el pacta sunt servanda y la buena fe. El primero dispone que los tratados son de obligatorio cumplimiento ${ }^{10}$, mientras que el segundo establece la forma en que los tratados deben ser cumplidos, esto es, de manera franca, honesta y leal, evitando en todo momento subterfugios o tomar ventaja indebida del acuerdo. A partir de estas dos premisas, se señalan en el artículo transcrito al menos cuatro reglas de interpretación, como son: la del sentido natural y ordinario de los términos, la del contexto, la del objeto y fin del tratado y la de la conducta ulterior de las partes. A continuación, precisamente, nos concentraremos en el desarrollo de esta última regla de interpretación.

\section{LA CONDUCTA ULTERIOR DE LAS PARTES COMO REGLA DE INTERPRETACIÓN PRINCIPAL}

A efectos de analizar en detalle esta regla general de interpretación de los tratados, es conveniente partir del art. 31, inciso 3, de la Convención de Viena de 1969 sobre el Derecho de los Tratados, el cual establece lo siguiente:

«Regla general de interpretación

3. Juntamente con el contexto, habrá de tenerse en cuenta:

a) Todo acuerdo ulterior entre las partes acerca de la interpretación del tratado o de la aplicación de sus disposiciones.

b) Toda práctica ulteriormente seguida en la aplicación del tratado por la cual conste el acuerdo de las partes acerca de la interpretación del tratado».

Con relación a esta regla de interpretación de los tratados, y teniendo en cuenta el Derecho internacional vigente, la doctrina y la jurisprudencia, es posible sostener lo siguiente.

\subsection{Regla general obligatoria}

Al estar contenida en el art. 31 de la Convención de Viena de 1969, la conducta ulterior de las partes debe ser entendida como una regla general de

\footnotetext{
9 Jiménez de Aréchaga, E., El Derecho Internacional Contemporáneo, Madrid, Tecnos, 1980, p. 55.

10 De Visscher, C., Problèmes d'interprétation judiciaire de Droit international Public, París, Pedone, 1963, p. 50.
} 
interpretación de aplicación obligatoria. Esto, a diferencia del art. 32 de ese mismo instrumento internacional, que señala el carácter discrecional de las reglas de interpretación ahí establecidas.

Esta afirmación se desprende claramente de la propia redacción del artículo antes citado, cuando en relación con las reglas que contiene señala que las mismas «habrán de tenerse en cuenta» por parte de los Estados parte, es decir, tienen carácter mandatorio, a diferencia del art. 32 que dispone que «se podrá acudir» a sus reglas, redacción que deja claramente a criterio de los intérpretes aplicarlas o no. A esta misma conclusión arriba la CDI en su Proyecto de conclusiones sobre los acuerdos ulteriores y la práctica ulterior de $2018^{11}$.

En consecuencia, se trata de un principio que debe ser empleado en todo proceso interpretativo, no quedando a discreción de las partes el emplearlo o no.

\subsection{Regla convencional y consuetudinaria}

La conducta ulterior de las partes, sin embargo, no solo tiene carácter vinculante por lo dispuesto por el art. 31 de la Convención de Viena de 1969, sino que también lo tiene por su carácter de norma consuetudinaria de alcance general ${ }^{12}$. Así lo ha señalado la abundante jurisprudencia internacional de diversos tribunales internacionales ${ }^{13}$; concretamente, la Corte Permanente de Arbitraje en el Asunto de la Reclamación rusa contra Turquía ${ }^{14}$; la Corte Permanente de Justicia Internacional en la opinión consultiva sobre la competencia de la OIT para la reglamentación internacional de las condiciones de trabajo de las personas empleadas en tareas agrícolas ${ }^{15}$; y la Corte Internacional de Justicia en el Asunto sobre la Diferencia territorial entre Libia y Chad, entre otros casos dictados posteriormente ${ }^{16}$. En el primero, la Corte sentenció:

«Según el Derecho internacional consuetudinario, que ha encontrado su expresión en el art. 31 de la Convención de Viena sobre Derecho de los Trata-

11 CDI, Proyecto de conclusiones 2018, cit., nota 5, pp. 18 y 21.

12 GardineR, R., Treaty Interpretation, Oxford, Oxford University Press, 2015, pp. 13 y ss.

13 NovaK, F., «Las reglas de interpretación de los tratados», en Torrez BERNÁRDEZ, S. et al. (coords.), El Derecho Internacional en el Mundo Multipolar del siglo XXI. Obra Homenaje al Profesor Luis Ignacio Sánchez Rodríguez, Madrid, Iprolex, 2013, p. 164.

14 De La Guardia, E., Derecho de los Tratados Internacionales, Buenos Aires, Editorial Ábaco de Rodolfo de Palma, 1997, p. 222.

15 Compétence de l'OIT pour la réglementation internationale des conditions du travail des personnes employées dans l'agriculture, avis consultatif, CPJI Série B, núm. 2, 1922, pp. 40-41.

${ }_{16}$ Plates-formes pétrolières (République islamique d'Iran c. Etats-Unis d'Amérique), Exception préliminaire, CIJ Recueil, 1996, p. 812; Ile de Kasikili/Sedudu (Botswana/Namibie), arrêt, CIJ Recueil, 1999, p. 18; LaGrand (Allemagne c. Etats-Unis d'Amérique), Fond, arrêt, CIJ Recueil, 2001, párr. 99; Souveraineté sur Pulau Ligitan et Pulau Sipadan (Indonésie/Malaisie), arrêt, CIJ Recueil, 2002, p. 645; Avena et autres ressortissants mexicains (Mexique c. Etats-Unis d'Amérique), arrêt, CIJ Recueil, 2004, p. 48; Conséquences juridiques de l'édification d'un mur dans le territoire palestinien occupé, avis consultatif, CIJ Recueil, 2004, p. 174; Application de la convention pour la prévention et la répression du crime de génocide (Bosnie-Herzégovine c. Serbie-et-Monténégro), arrêt, CIJ Recueil, 2007, párr. 160. 
dos, un tratado debe ser interpretado de buena fe conforme al sentido ordinario que haya de atribuir a sus términos en su contexto y a la luz de su objeto y fin» ${ }^{17}$.

En el mismo sentido se ha pronunciado el Tribunal Europeo de Derechos Humanos en el Asunto Golder c. United Kingdom ${ }^{18}$, la Corte Interamericana de Derechos Humanos en su opinión consultiva sobre la expresión «leyes» en el art. 30 de la Convención Americana sobre Derechos Humanos ${ }^{19}$, y el Órgano de Apelación de la Organización Mundial del Comercio (OMC) en el Asunto Estados Unidos-Pautas para la gasolina reformulada y convencional ${ }^{20}$.

Por tanto, el carácter general y obligatorio de este principio de interpretación de los tratados tiene un doble sustento normativo en el Derecho internacional: convencional y consuetudinario, resultando en consecuencia de aplicación a todos los Estados, independientemente de que sean o no partes de la Convención de Viena de 1969.

\subsection{Regla aplicable en forma combinada}

De otro lado, la conducta ulterior de las partes no puede ser empleada para la interpretación de un tratado de manera aislada, sino que debe ser aplicada como regla de interpretación en conjunto con las demás reglas que el art. 31 contiene; a saber, el sentido ordinario y natural de los términos, el contexto y la conformidad con el objeto y fin del tratado.

El empleo de la expresión «Regla general de interpretación» en singular y no en plural para encabezar el art. 31 de la Convención de Viena tenía precisamente el propósito de dar a entender a estas cuatro reglas como una fórmula combinada, en el sentido de ser aplicadas en conjunto y no de manera aislada ${ }^{21}$.

El proceso de interpretación constituye así una unidad y las disposiciones del artículo forman una regla única con partes íntimamente ligadas entre sí $^{22}$. En este sentido, todas las reglas consagradas en dicho artículo deben

17 Différend territorial (Jamahiriya arabe libyenne/Tchad), arrêt, CIJ Recueil, 1994, p. 22.

18 Golder c. United Kingdom, Série A, núm. 18, TEDH 1975.

19 Opinión Consultiva sobre la expresión «leyes» en el artículo 30 de la Convención Americana sobre Derechos Humanos, OC 6/86, 9 de mayo de 1986, párr. 13. También véase la OC 1/82, 24 de septiembre de 1982, p. 14: «Para la interpretación del artículo 64 de la Convención, la Corte utilizará los métodos tradicionales de Derecho internacional, tanto en lo que se refiere a las reglas generales de interpretación, como en lo que toca a los medios complementarios, en los términos en que los mismos han sido recogidos por los arts. 31 y 32 de la Convención de Viena sobre Derecho de los Tratados».

${ }_{20}$ Asunto Estados Unidos-Pautas para la gasolina reformulada y convencional, OMC. Informe del Grupo, WT/DS2/R, 29 de enero de 1996, pp. 21-23. Véase NolTE, G., "Jurisprudence under special regimes relating to subsequent agreements and subsequent practice: Second report for the ILC Study Group on treaties over time», en Nolte, G. (ed.). Treaties and Subsequent Practice, Oxford, Oxford University Press, 2013, pp. 210-305, p. 215.

21 CDI, Anuario, 1966, vol. II, Documento A/6309/Rev.1, pp. 218 y 220.

22 Véase González Campos, J., Sánchez Rodríguez, L. I. y Andrés Sáenz de Santa María, P., Curso de Derecho Internacional Público, Navarra, Thomson-Civitas, 2008, p. 366. 
ser consideradas y todas tienen igual valor, no dominando una sobre las otras $^{23}$.

\subsection{Contenido y carácter de prueba objetiva}

La conducta ulterior de las partes como regla general establece que las disposiciones de un tratado deben ser interpretadas de conformidad con la interpretación acordada por todas las partes de manera expresa (interpretación auténtica expresa) o con la aplicación práctica del tratado realizada en común por los Estados parte (interpretación auténtica tácita). En otras palabras, la conducta ulterior de las partes tiene un doble contenido, pues incluye no solo el acuerdo expreso, sino también la práctica ulterior seguida en la aplicación del tratado por todas las partes en él, debiendo entenderse ambas como una manifestación clara de esta regla general de interpretación.

Asimismo, tanto el acuerdo expreso como la práctica de los Estados deberán ser reconocidos como una prueba objetiva respecto del verdadero sentido $\mathrm{y}$ alcance del acuerdo existente entre las partes ${ }^{24}$.

Prueba objetiva, sin embargo, no es sinónimo de prueba definitiva. Así lo precisa la CDI, en su Proyecto de conclusiones sobre los acuerdos ulteriores y la práctica ulterior de 2018, al afirmar:

«La caracterización como "medios auténticos de interpretación” de los acuerdos ulteriores y la práctica ulteriormente seguida por las partes en virtud del artículo 31, párrafo 3.a) y b), no implica que esos medios tengan necesariamente efectos concluyentes.

[...] pese a lo que dan a entender algunos autores, los acuerdos ulteriores y la práctica ulterior por la que conste el acuerdo de las partes acerca de la interpretación del tratado no tienen que ser jurídicamente vinculantes. Esto se confirma en el proyecto de conclusión 10, párrafo 1. Así pues, cuando la Comisión señaló que un "acuerdo ulterior" constituía "una interpretación auténtica por las partes que debe ser tenida en cuenta a efectos de la interpretación del tratado", no fue tan lejos como para afirmar que esa interpretación fuera necesariamente concluyente en el sentido de prevalecer sobre los demás medios de interpretación» ${ }^{25}$.

${ }^{23}$ Villiger, M., Commentary on the 1969 Vienna Convention on the Law of Treaties, Leiden/Boston, Martinus Nijhoff, 2009, p. 435. Sin embargo, la práctica de algunos tribunales nos indica un comportamiento ambivalente. Véase TORRES BERnÁRdEZ, S., "Interpretation of Treaties by the International Court of Justice following the adoption of the Vienna Convention on the Law of Treaties», en HAFNer, G., Loibl, G., Rest, A., Sucharipa-Behrmann, L. y ZemaneK, K. (eds.), Liber Amicorum Professor Ignaz. Seidl-Hohenveldern in honour of his $80^{\text {th }}$ Birthday, La Haya, Kluwer Law International, 1998, pp. 721-739, esp. pp. 721 y ss. Asimismo, VILliger, M., «The rules on interpretation: misgivings, misunderstandings, miscarriage? The 'crucible' intended by the International Law Commission", en CANNIZARo, E. (ed.), The Law of Treaties. Beyond the Vienna Convention, Oxford, Oxford University Press, 2011, pp. 105-122, esp. pp. 114-116; y Nolte, G., "Subsequent practice as a means of interpretation in the jurisprudence of the WTO Apellate Body», en la misma obra, pp. 138-144, esp. pp. 139-141.

${ }_{24}$ CDI, Anuario, 1966, cit., nota 21, p. 243.

25 CDI, Proyecto de conclusiones 2018, cit., nota 4, p. 25. 
Por tanto, el hecho de que la conducta ulterior de las partes sea una regla de aplicación obligatoria en la interpretación de un tratado, y que la misma constituya prueba objetiva de la voluntad de las partes, no implica que tal interpretación sea definitiva, o que no pueda ser contradicha al aplicar otros principios de interpretación de los tratados. Esto se deriva de la ausencia de jerarquía entre los principios de interpretación de los tratados, como también del carácter combinado de su aplicación.

\subsection{El término «ulterior»}

¿Qué acuerdos y prácticas deben considerarse como «ulteriores» a un tratado? La Convención de Viena sobre el Derecho de los Tratados de 1969 no define qué debe entenderse por «ulterior». La CDI ha optado por otorgar el carácter de ulterior a una conducta (acuerdo o práctica) en un punto posterior a la «celebración» del tratado.

Sin embargo, también puede existir dificultad al identificar el momento de «celebración» de un tratado, en tanto no existe un solo procedimiento en relación con las etapas que siguen al fin de las negociaciones de un tratado y su entrada en vigor ${ }^{26}$. Así, podría considerarse que la firma es el momento de celebración del tratado, por lo que los acuerdos y prácticas posteriores a tal hito serían «ulteriores» en el sentido del art. 31 (3) (a) y (b). En sentido opuesto, podría argüirse que también se debe incluir la etapa en la que se depositan los instrumentos de ratificación, por ejemplo, como parte de la celebración de cierto tratado, con lo cual su entrada en vigor sería el momento desde el cual el tratado ha sido celebrado. Consecuentemente, solo la conducta posterior a la entrada en vigor sería «ulterior».

Al respecto, existe la postura según la cual es preferible acudir a los medios de interpretación solo después de que el tratado ha entrado en vigor, en tanto solo desde ese punto es posible hablar de la existencia de una práctica en la aplicación de un tratado, ya que un tratado no se aplica antes de su entrada en vigor ${ }^{27}$.

Sin embargo, la CDI ha considerado que la celebración es, con frecuencia, anterior al momento en que el tratado entra en vigor, ya que muchos tratados pueden considerarse celebrados («concluded») antes de su entrada en vigor efectiva. En ese sentido, se indica que, para los propósitos de la materia, «celebración» es cuando el texto del tratado ha quedado establecido como auténtico y definitivo en el sentido del art. 10 de la Convención de Viena. Ello —indica la CDI—en tanto es después de la celebración del tratado - y no solo después de su entrada en vigor-que los acuerdos ulteriores y la práctica ulterior pueden ocurrir ${ }^{28}$.

\footnotetext{
${ }^{26}$ Gardiner, R., «The Vienna Convention Rules on Treaty Interpretation», en Hollis, D. (ed.) The Oxford Guide to Treaties, Oxford, Oxford University Press, 2012, pp. 475-506, esp. p. 484.

27 DJefFal, C., Static and Evolutive Treaty Interpretation, Cambridge, Cambridge University Press, 2015, p. 165.

28 CDI, Proyecto de conclusiones 2018, cit., nota 5, p. 27.
} 


\subsection{Alcances del acuerdo ulterior}

En cuanto al acuerdo ulterior, como primer componente o manifestación de la conducta ulterior, la CDI lo define en su conclusión 4 del Proyecto de 2018 como «un acuerdo acerca de la interpretación del tratado o la aplicación de sus disposiciones al que hayan llegado las partes después de la celebración del tratado». Además añade en su conclusión 10 (1) que este acuerdo «requiere un entendimiento común acerca de la interpretación de un tratado que las partes reconozcan y acepten ${ }^{29}$.

Esta definición alude entonces, en primer lugar, a un acuerdo en el que deben participar todas las partes en un tratado, después de su celebración, respecto de la interpretación o aplicación de una o más de sus disposiciones. Al hacer referencia a un entendimiento común, la CDI está aludiendo a que el acuerdo ulterior debe basarse en el consenso de todas las partes del tratado que viene siendo interpretado, pues de haber divergencias, no se podría sostener la existencia de tal acuerdo, tal como lo señaló el tribunal arbitral que resolvió el caso German External Debts ${ }^{30}$.

Asimismo, este acuerdo puede consistir en un nuevo tratado o en un acuerdo internacional sin carácter vinculante, como podría ser el caso de un memorando de entendimiento ${ }^{31}$. Esto último ha sido confirmado repetidamente por la jurisprudencia internacional ${ }^{32}$, pero también por la CDI en su conclusión 10 (1) del Proyecto de 2018, al disponer: «Dicho acuerdo podrá ser legalmente vinculante, aunque no tendrá que serlo, para ser tenido en cuenta» ${ }^{33}$.

Incluso el acuerdo ulterior podría consistir en un acuerdo verbal, según se sostuvo ante la Corte Internacional de Justicia en el Asunto fábricas de celulosa en el río Uruguay ${ }^{34}$.

También pueden tratarse de actos separados que combinados reflejen una voluntad común; como lo señala la CDI, el acuerdo "presupone un acto o compromiso común deliberado de las partes, incluso si este consiste en actos individuales por los cuales estas manifiestan su entendimiento común acerca de la interpretación del tratado o la aplicación de sus disposiciones» ${ }^{35}$.

De otro lado, aquellos acuerdos que sean concertados antes de la celebración del tratado o en el momento de la celebración, se considerarán como

\footnotetext{
29 Ibid., pp. 28 y 81.

30 Ibid., p. 81.

31 Ibid., pp. 30 y 53. Véase también GARDINER, R., op. cit., nota 12, p. 244.

32 Linderfalk, U., The modern international law as expressed in the 1969 Vienna Convention on the Law of Treaties, Dordrecht, The Netherlands, Springer, 2007, p. 163.

33 CDI, Proyecto de conclusiones 2018, cit., nota 5, p. 27.

${ }^{34}$ Usines de pâte à papier sur le fleuve Uruguay (Argentine c. Uruguay), mesures conservatoires, CIJ Recueil, 2006, pp. 113 y ss.

35 CDI, Proyecto de conclusiones 2018, cit., nota 5, p. 32.
} 
parte del tratado ${ }^{36}$. Así lo afirma la CDI, pero también la Corte Internacional de Justicia, en el Asunto Ambatielos, donde señaló: «Las disposiciones de la Declaración tienen el carácter de cláusula de interpretación y, como tales, deben ser consideradas como parte integrante del tratado» ${ }^{37}$. Del mismo modo, los acuerdos que se concierten entre las partes con posterioridad al tratado constituirán una interpretación auténtica del mismo. Esto fue lo que se señaló en el Asunto de la Indemnización rusa al afirmarse: «El cumplimiento de las obligaciones es, entre Estados, como entre particulares, el comentario más seguro sobre el sentido de esas obligaciones» ${ }^{38}$.

Finalmente, se debe apuntar que el acuerdo ulterior constituye ipso facto un medio de interpretación del tratado, a diferencia de la práctica ulterior, que para convertirse en un medio auténtico de interpretación requiere que la misma ponga de manifiesto el acuerdo de las partes sobre el verdadero sentido y alcance del tratado ${ }^{39}$.

\subsection{Alcances de la práctica ulterior}

En cuanto a la práctica ulterior, como segundo componente o manifestación de la regla de la conducta ulterior, la CDI la define en su conclusión 4 como «el comportamiento observado en la aplicación del tratado, después de su celebración, por el cual conste el acuerdo de las partes acerca de la interpretación del tratado» ${ }^{40}$.

La práctica ulterior como método de interpretación fue empleado por la Corte Permanente de Justicia Internacional en su opinión consultiva sobre la competencia de la OIT para la reglamentación internacional de las condiciones de trabajo de las personas empleadas en tareas agrícolas, donde indicó que «si hubiera alguna ambigüedad, la Corte podrá, con el fin de determinar el verdadero sentido, examinar la forma en que se ha aplicado el tratado» ${ }^{41}$. Lo mismo fue apuntado por este tribunal en el Asunto de los empréstitos brasileños ${ }^{42}$ y luego, la Corte Internacional de Justicia apeló a este mismo principio en el Asunto Corfú para señalar «la actitud ulterior de las partes demuestra que, al participar en el Convenio especial, no tenían la intención de impedir que la Corte fijase el importe de la indemnización» ${ }^{43}$.

\footnotetext{
36 ONU, Conferencia de las Naciones Unidas sobre el Derecho de los Tratados, Periodos de sesiones primero y segundo, Viena, 26 de marzo-24 de mayo de 1968 y 9 de abril-22 de mayo de 1969, Documentos oficiales, Documentos de la Conferencia, Nueva York, 1971, p. 44.

37 Ambatielos (Grèce c. Royaume-Uni), Exception préliminaire, CIJ Recueil, 1952, p. 44.

38 Corte Permanente de Arbitraje, Asunto de la indemnización rusa, Reports of International Arbitral Awards, vol. XI, 1912, p. 433.

39 CDI, Proyecto de conclusiones 2018, cit., nota 5, p. 31.

40 Ibid., p. 28.

${ }^{41}$ Compétence de l'OIT pour la réglementation internationale des conditions du travail des personnes employées dans l'agriculture, avis consultatif, CPJI, Série B, núm. 2, 1922, p. 39.

42 Emprunts brésiliens, arrêt, CPJI, Série A, núm. 21, 1929, p. 119.

43 Détroit de Corfou (Royaume-Uni de Grande-Bretagne et d'Irlande du Nord c. Albanie), Fond, arrêt, CIJ Recueil, 1949, p. 25.
} 
A lo anterior podría añadirse la práctica ulterior identificada en la opinión consultiva sobre Namibia ${ }^{44}$ :

«Un clásico y comúnmente citado ejemplo de práctica ulterior es el referido a la forma en que los miembros de las Naciones Unidas han interpretado el artículo 27 (3) de la Carta de las Naciones Unidas, relativo a la votación por los miembros del Consejo de Seguridad. Al respecto, la Corte Internacional de Justicia ${ }^{45}$ confirmó la práctica seguida por el Consejo de Seguridad desde 1946 en el sentido de que la abstención de un miembro permanente no impide la adopción de una resolución. Ello, a pesar de que, de una primera lectura, el artículo pareciera haber exigido el voto afirmativo y no solo la abstención de los miembros permanentes» ${ }^{46}$.

La práctica alude entonces a toda conducta o comportamiento desarrollado por todas las partes en un tratado que permite identificar un entendimiento sobre la forma en que debe ser aplicado o interpretado dicho instrumento.

La esencia de esta regla es que debemos estar frente a la aplicación o implementación sistemática o repetida de un tratado, sea a través de una práctica concordante de todas las partes (donde estas realizan básicamente lo mismo en la ejecución del acuerdo), o mediante una conducta unilateral de ejecución que haya sido aceptada por todas las otras partes del acuerdo (aquiescencia) ${ }^{47}$.

Por tanto, cuando hablamos de comportamiento aludimos no solo a acciones sino también a omisiones o incluso a silencios que contribuyan a conformar el acuerdo ${ }^{48}$. Sobre esto último, es decir, respecto a la posibilidad de que la práctica ulterior se pueda conformar a partir de silencio, resulta pertinente citar la conclusión 10 (2) del Proyecto de la CDI de 2018, la cual señala: «El silencio de una o más partes puede constituir aceptación de la práctica ulterior cuando las circunstancias requieran alguna reacción». Esto ha sido repetidamente confirmado por la doctrina ${ }^{49}$ y la jurisprudencia internacional, en particular por la Corte Internacional de Justicia en numerosos fallos, como sucedió en el Asunto del Templo de Preah Vihear ${ }^{50}$ o en el de las

${ }^{44}$ Aust, A., Modern Treaty Law and Practice, Cambridge, Cambridge University Press, 2013, pp. 215-216; y Kolb, R., The Law of Treaties: An Introduction, Cheltenham, Edward Elgar Publishing, 2016, p. 144.

${ }_{45}$ Conséquences juridiques pour les États de la présence continue de l'Afrique du Sud en Namibie (Sud-Ouest africain) nonobstant la résolution 276 (1970) du Conseil de Sécurité, avis consultatif, CIJ Recueil, 1971, p. 22.

46 La frase interpretada proviene del texto en inglés de la Carta de las Naciones Unidas: «The concurring votes of all permanent members». Carta de las Naciones Unidas, aprobada en la Conferencia de San Francisco el 26 de junio de 1945. Véase https://www.un.org/es/charter-united-nations/. El término «concurring» sugería que los miembros del Consejo de Seguridad debían otorgar su voto afirmativo; sin embargo, en la práctica se optó por no darle tal sentido.

47 NovaK, F., op. cit., nota 13, p. 163.

48 CDI, Anuario, 1964, vol. II, Documento A/CN.4/167 y Add.1 a 3, p. 60.

49 Entre los que destaca GARDINER, R., op. cit., nota 12, p. 267; YASSEN, M., op. cit., nota 1, pp. 1-114, esp. p. 49; entre otros.

50 Temple de Préah Vihéar (Cambodge c. Thaïlande), Fond, arrêt, CIJ Recueil, 1962, p. 23. 
Plataformas petroleras entre la República Islámica de Irán y los Estados Unidos de América ${ }^{51}$.

Este comportamiento a su vez, puede presentarse, según la CDI, en los actos oficiales que desarrolle un Estado a nivel internacional e interno que sirvan para aplicar un tratado, tales como declaraciones oficiales, declaraciones dadas en una conferencia internacional, declaraciones dadas en un litigio internacional o en el seno de una organización internacional, como a través de la aprobación de legislación nacional, la adopción de medidas administrativas de implementación, sentencias dictadas en el ámbito interno, etc. Sobre las sentencias dictadas por tribunales internos como manifestación de una práctica ulterior, cabe citar el caso Jelisić, donde el Tribunal Penal Internacional para la ex-Yugoslavia señaló:

«La Sala de Primera Instancia ha tenido en cuenta también la práctica ulterior basada en la Convención. Se ha atribuido especial importancia a las sentencias dictadas por el Tribunal para Rwanda [...]. También se han tenido en cuenta la práctica de los Estados, particularmente por medio de sus órganos jurisdiccionales internos y la labor en esa esfera realizada por los órganos internacionales» ${ }^{52}$.

La práctica ulterior desarrollada por una organización internacional, también puede ser útil para interpretar su tratado constitutivo. Esto lo reconoce la CDI en su Proyecto de 2018, cuando en su conclusión 12 (3) afirma que: «La práctica de una organización internacional en la aplicación de su instrumento constitutivo puede contribuir a la interpretación de dicho instrumento». Nótese que se refiere a la práctica de la organización misma y no a la de los Estados miembros; incluso podría tratarse de una práctica que no tenga la aceptación de los Estados miembros ${ }^{53}$.

En este punto resulta interesante citar la opinión del juez Hersch Lauterpacht dada en la opinión consultiva de la Corte Internacional de Justicia sobre el África sudoccidental, donde dijo que la interpretación:

«adecuada de un instrumento constitutivo debe tener en cuenta no solo el sentido estrictamente literal del instrumento original, sino también su funcionamiento efectivo en la práctica y a la luz de las tendencias observadas en la vida de la Organización ${ }^{54}$.

La Corte Internacional de Justicia lo ha reconocido también en su opinión consultiva sobre la legalidad del uso por los Estados de armas nucleares en conflictos armados, al señalar que «los instrumentos constitutivos de las organizaciones internacionales son tratados multilaterales, a los que se apli-

\footnotetext{
51 Plates-formes pétrolières (République islamique d'Iran c. Etats-Unis d'Amérique), Exception préliminaire, CIJ Recueil, 1996, p. 815.

52 Tribunal Penal Internacional de la ex-Yugoslavia, Sala de Primera Instancia, Prosecutor v. Goran Jelisić, 1999, IT-95-10-T, párr. 61.

53 CDI, Proyecto de conclusiones 2018, cit., nota 5, pp. 109-110.

54 Procédure de vote applicable aux questions touchant les rapports et pétitions relatifs au Territoire du Sud-Ouest africain, avis consultatif, CIJ Recueil, 1955, p. 106.
} 
can las normas bien establecidas de interpretación de los tratados», y luego agregar que ello incluye «toda práctica ulteriormente seguida en la aplicación del tratado por la cual conste el acuerdo de las partes acerca de la interpretación del tratado" ${ }^{55}$. Pero también cabe destacar especialmente el Asunto de la frontera terrestre y marítima entre Camerún y Nigeria, donde esta misma Corte concluyó:

«Del análisis de los textos de los tratados y de la práctica realizada en los párrafos 64 y 65 supra se desprende que la Comisión de la Cuenca del Lago Chad es una organización internacional que ejerce sus competencias dentro de una zona geográfica determinada; que, no obstante, su objeto no es resolver, en el plano regional, los asuntos relacionados con el mantenimiento de la paz y la seguridad internacionales y, por tanto, no entra dentro del Capítulo VII de la Carta ${ }^{56}$.

De otro lado, en relación a la práctica ulterior se debe precisar que la conducta que deberá ser tomada en cuenta para efectos de servir como medio de interpretación será aquella que se desarrolle de buena fe, es decir, «una mala aplicación manifiesta del tratado, a diferencia de una aplicación de buena fe (aunque sea errónea), no es, por tanto, una "aplicación del tratado" en el sentido de los arts. 31 y $32{ }^{57}$.

Con respecto a si la práctica ulterior debe consistir en un comportamiento repetido de manera frecuente y uniforme o puede tratarse de una aplicación dada una sola vez ${ }^{58}$, se suele citar el informe del órgano de apelación de la OMC dado en el Asunto Japón-Impuestos sobre las bebidas alcohólicas, donde se estableció:

«Generalmente, en Derecho internacional, la esencia de la práctica ulterior en la interpretación de un tratado ha sido reconocida como una secuencia de actos o pronunciamientos concordantes, comunes y consistentes, [...] que implican el acuerdo existente entre las partes respecto a su interpretación. Un acto aislado no es generalmente suficiente para establecer una práctica subsecuente; esta es una secuencia de actos que establecen el acuerdo de las partes de forma relevante» ${ }^{59}$.

De otra parte, respecto a quiénes pueden realizar la práctica ulterior, se debe señalar que esta puede consistir en actos del Poder Ejecutivo, del Poder Legislativo o del Poder Judicial de los Estados parte, donde se verifique la in-

${ }_{55}$ Licéité de l'utilisation des armes nucléaires par un Etat dans un conflit armé, avis consultatif, CIJ Recueil, 1996, pp. 66, 74 y 75.

56 Frontière terrestre et maritime entre le Cameroun et le Nigéria (Cameroun c. Nigéria ; Guinée Équatoriale (intervenant)), Exceptions préliminaires, CIJ Recueil, 1998, pp. 306-307, citado en CDI, Proyecto de conclusiones 2018, cit., nota 5, p. 103.

57 CDI, Proyecto de conclusiones 2018, cit., nota 5, p. 34.

58 Villiger, M., Commentary..., op. cit., nota 23, p. 431; LinderfalK, U., The modern international, op. cit., nota 2, p. 166.

59 Asunto Japón-Impuestos sobre las Bebidas Alcohólicas, AB-1996-2, 4 de octubre de 1996, pp. 1213. Véase GARDINER, R., op. cit., nota 12, pp. 227-228. En sentido contrario se manifiesta la CDI, para el caso del art. 32 de la Convención de Viena: «Si el concepto de práctica ulterior como medio de interpretación de los tratados se distingue de un posible acuerdo entre las partes, la frecuencia no es un elemento necesario de la definición del concepto de "práctica ulterior" en sentido amplio (en virtud del artículo 32)», CDI, Proyecto de conclusiones 2018, cit., nota 5, p. 39. 
terpretación de una o más disposiciones del tratado a través de su ejecución o implementación. Así lo confirma la conclusión 5 del Proyecto de conclusiones de la CDI de 2018:

«1. La práctica ulterior en virtud de los artículos 31 y 32 puede consistir en cualquier comportamiento de una parte en la aplicación de un tratado, ya sea en el ejercicio de sus funciones ejecutivas, legislativas, judiciales o de otra índole.

2. Todo otro comportamiento, incluido el de actores no estatales, no constituye práctica ulterior en virtud de los artículos 31 y 32. No obstante, dicho comportamiento puede ser pertinente al evaluar la práctica ulterior de las partes en un tratado».

Y es que la aplicación del tratado corresponde generalmente a las autoridades del Estado. Como lo señaló el Tribunal de Reclamaciones Irán-Estados Unidos:

«Según un principio reconocido de la interpretación de los tratados, hay que tener en cuenta, junto con el contexto, toda práctica ulterior seguida en la aplicación de un tratado internacional. No obstante, esa práctica ha de ser una práctica de las partes en el tratado por la cual conste el acuerdo de las partes acerca de la interpretación del tratado.

Si bien uno de los participantes en las negociaciones para llegar a un acuerdo, a saber, el Banco Markazi, es una entidad del Irán y, por ello, su práctica puede atribuirse al Irán por ser una de las partes en las declaraciones de Argel, los otros participantes en las negociaciones para llegar a un acuerdo y en los acuerdos en sí, a saber, los bancos de los Estados Unidos, no son entidades del Gobierno de los Estados Unidos y su práctica no puede ser atribuida a los Estados Unidos en calidad de otra de las partes en las declaraciones de Argel ${ }^{60}$.

En esta línea, se debe precisar que la jerarquía del órgano estatal que lleve adelante la práctica ulterior será irrelevante, pues lo que importa es la realización por este de una función estatal; esto fue reconocido por la Corte Internacional de Justicia en el Asunto de los derechos de los nacionales de los Estados Unidos de América en Marruecos ${ }^{61}$. Puede tratarse entonces de una autoridad de inferior jerarquía, pues si el gobierno está al tanto de su práctica y no la contradice en un plazo razonable, deberá asumirse como una práctica ulterior del Estado.

Lo anterior no excluye, por supuesto, comprender dentro de la práctica ulterior el comportamiento de actores privados a quienes se les delegue autoridad o funciones públicas. Sin embargo, no están comprendidos comportamientos de Estados u Organizaciones Internacionales que no sean parte del tratado, de órganos técnicos de asesoramiento en un tratado, etc., lo que no niega que, en algunos casos, existan algunos actores no estatales cuya práctica deba ser tomada en cuenta al evaluar la práctica ulterior de las partes en un tratado. Es el caso del Comité Internacional de la Cruz Roja (CICR),

${ }^{60}$ Estados Unidos de América y otros v. República Islámica de Irán y otros, Laudo núm. 108-A16/582/591-FT, Iran-United States Claims Tribunal Reports, vol. 5, 1984, pp. 57 y ss.

61 Droits des ressortissants des États-Unis d'Amérique au Maroc (France c. États-Unis d'Amérique), arrêt, CIJ Recueil, 1952, pp. 176 y ss. 
que ocasionalmente brinda interpretaciones sobre los Convenios de Ginebra de $1949^{62}$ y sus Protocolos de $1977^{63}$, por mandato de su propio Estatuto, o del Monitor de Minas Terrestres y Municiones en Racimo respecto de la Convención sobre la prohibición del empleo, almacenamiento, producción y transferencia de minas antipersonal y sobre su destrucción de 1997 (Convención de Ottawa ${ }^{64}$ y la Convención sobre municiones en racimo de $2008{ }^{65}$ (Convención de Oslo) ${ }^{66}$.

En cuanto al número de Estados que deben participar de la práctica, a efectos de que esta tenga valor interpretativo, claramente son todas las partes del tratado a interpretar. Al respecto precisa la CDI:

«En el texto que se aprobó provisionalmente en 1964 se hablaba de una práctica que "evidencie [...], una interpretación concorde de todas las partes". Al suprimir el término "todas", la Comisión no se proponía modificar la norma. Estimó que la expresión "el acuerdo de las partes" se refería necesariamente a todas las partes. Omitió la palabra "todas" simplemente para evitar cualquier posibilidad de que se entendiese erróneamente que todas y cada una de las partes debían haber seguido la práctica, cuando basta que la hayan aceptado» ${ }^{67}$.

En todo caso, si la práctica no contara con la aceptación de todas las partes, pero sí de una mayoría o incluso de unas pocas, podrá servir como medio complementario de interpretación, esto es, dentro de los alcances del art. 32 de la Convención de Viena de 1969.

\subsection{Diferencias entre los acuerdos ulteriores y la práctica ulterior}

Al respecto, según Rahim Moloo, existe un «evidenciary continuum», en el sentido de que si bien toda la conducta ulterior requiere de un alto estándar de prueba («is highly probative»), el umbral de prueba es más alto en el caso de los acuerdos ulteriores en comparación con la práctica ulterior ${ }^{68}$.

Una segunda distinción tiene que ver con qué actores realizan una conducta relevante para los propósitos de los acuerdos ulteriores y la práctica ulterior. En el caso de la práctica ulterior, como se desarrolla posteriormente en este trabajo, el comportamiento puede ser realizado por todos aquellos fun-

\footnotetext{
${ }^{62}$ Convenios de Ginebra del 12 de agosto de 1949. Véase https:/www.icrc.org/es/doc/assets/files/ publications/convenios-gva-esp-2012.pdf.

63 Protocolos Adicionales I y II de los Convenios de Ginebra del 8 de junio de 1977. Véase https:// www.icrc.org/es/doc/resources/documents/misc/additional-protocols-1977.htm.

64 Convención sobre la Prohibición del Empleo, Almacenamiento, Producción y Transferencia de Minas Antipersonal y sobre su Destrucción del 18 de septiembre de 1997. Véase https://www.un.org/es/ disarmament/instruments/convention_landmines.shtml.

${ }_{65}$ Convención sobre Municiones en Racimo del 30 de mayo de 2008. Véase https://www.un.org/es/ disarmament/conventionalarms/convention/clustermunitions.shtml.

66 CDI, Proyecto de conclusiones 2018, cit., nota 5, pp. 40-44.

67 ONU, cit., nota 46, p. 44.

68 Moloo, R., "When Actions Speak Louder than Words: The Relevance of Subsequent Party Conduct to Treaty Interpretation», Berkeley Journal of International Law, vol. 31, 2013, núm. 1, pp. 39-87, pp. 57-58.
} 
cionarios del Estado que apliquen el tratado. En contraste, los actores cuya conducta será relevante para la configuración de acuerdos ulteriores serán, generalmente, autoridades del gobierno autorizados a interpretar tratados ${ }^{69}$.

\subsection{Las conferencias de Estados parte}

Respecto de las conferencias de Estados parte en un tratado como manifestación de acuerdos o prácticas ulteriores, la CDI le dedica la conclusión 11 dentro de su Proyecto de 2018, donde establece:

«1. Una conferencia de Estados parte, en el sentido del presente proyecto de conclusiones, es una reunión de partes en un tratado a los fines de examinar o aplicar el tratado, salvo cuando actúen en calidad de miembros de un órgano de una organización internacional.

2. El efecto jurídico de una decisión adoptada en el marco de una conferencia de Estados parte depende principalmente del tratado y, en su caso, del reglamento aplicable. Dependiendo de las circunstancias, dicha decisión puede constituir, explícita o implícitamente, un acuerdo ulterior en virtud del artículo 31, párrafo 3.a), o dar lugar a una práctica ulterior en virtud del artículo 31, párrafo 3.b), o a una práctica ulterior en virtud del artículo 32. Las decisiones adoptadas en el marco de una conferencia de Estados parte a menudo ofrecen una diversidad no excluyente de opciones prácticas para la aplicación del tratado».

La conclusión antes citada se explica en la medida que este tipo de conferencias, celebradas periódicamente y en las que participan representantes de todos los Estados parte de un tratado, suelen precisamente estar dedicadas al análisis de la buena marcha, aplicación e implementación del instrumento internacional al que se encuentran sujetos, por lo cual muchas veces desarrollan acuerdos de interpretación o dan lugar a prácticas que implican una interpretación consensuada del tratado.

Este es el caso, por ejemplo, de la Conferencia de las Partes de la Convención Marco de las Naciones Unidas sobre el Cambio Climático de $1992^{70}$ o del Protocolo de Kyoto de $1997^{71}$, o del Estatuto de Roma de $1998^{72}$ que creó la Corte Penal Internacional ${ }^{73}$. La forma que suelen adoptar estas interpretaciones varía de un tratado a otro, denominándose en algunos casos resoluciones, y en otros directrices, decisiones, etc.

69 Ibid., p. 69.

70 Convención Marco de las Naciones Unidas sobre el Cambio Climático del 9 de mayo de 1992. Véase https://unfccc.int/resource/docs/convkp/convsp.pdf. NAMIHAS, S. (ed.), Conferencia sobre Cambio Climático. COP 20, Lima, Instituto de Estudios Internacionales (IDEI) de la Pontificia Universidad Católica del Perú, Sociedad Peruana de Derecho Ambiental (SPDA) y Fundación Konrad Adenauer, 2014.

71 Protocolo de Kyoto de la Convención Marco de las Naciones Unidas sobre el Cambio Climático del 11 de diciembre de 1997. Véase https://unfccc.int/resource/docs/convkp/kpspan.pdf.

72 Estatuto de Roma de la Corte Penal Internacional del 17 de julio de 1998. Véase https://www. un.org/spanish/law/icc/statute/spanish/rome_statute(s).pdf.

73 Novak, F. y García-Corrochano, L., Derecho Internacional Público, Tomo 3: Otros Sujetos de Derecho Internacional y Solución de Controversias, Lima, Thomson \& Reuters, 2018, p. 257. 
En todo caso, es pertinente precisar que la competencia de este tipo de conferencias para llevar a cabo acuerdos o prácticas interpretativas puede estar explícitamente contemplada en el tratado materia de interpretación o simplemente las partes la pueden presumir como una competencia implícita siempre, claro está, que sean todas las partes en común las que efectúen dicha interpretación.

\subsection{Los órganos de expertos}

Con relación a los órganos de expertos creados por tratados, la conclusión 13 (3) del Proyecto de 2018 de la CDI dispone lo siguiente:

«El pronunciamiento de un órgano de expertos creado en virtud de un tratado puede dar lugar, o referirse, a un acuerdo ulterior o una práctica ulterior de las partes en virtud del artículo 31, párrafo 3, o a una práctica ulterior en virtud del artículo 32. No se presumirá que el silencio de una parte constituye una práctica ulterior en virtud del artículo 31, párrafo 3.b), por la que se acepta una interpretación de un tratado expresada en un pronunciamiento de un órgano de expertos creado en virtud de un tratado».

Efectivamente, en la práctica convencional, diversos tratados crean órganos de expertos que actúan de manera autónoma con el propósito de vigilar, pero también apoyar, la aplicación e implementación del referido instrumento. La CDI señala, como ejemplos, la Junta Internacional de Fiscalización de Estupefacientes creada de acuerdo con la Convención Única sobre Estupefacientes de 1961; el Comité para la Eliminación de la Discriminación Racial, creado por la Convención Internacional sobre la Eliminación de todas las Formas de Discriminación Racial de 1966; el Comité de Derechos Humanos, creado por el Pacto Internacional de Derechos Civiles y Políticos de 1966; el Comité para la Eliminación de la Discriminación contra la Mujer, creado por la Convención sobre la Eliminación de todas las Formas de Discriminación contra la Mujer de 1979; la Comisión de Límites de la Plataforma Continental, establecida en virtud de la Convención de las Naciones Unidas sobre el Derecho del Mar de 1982; el Comité contra la Tortura, creado por la Convención contra la Tortura y otros Tratos o Penas Crueles, Inhumanos o Degradantes de 1984; el Comité de los Derechos del Niño, creado por la Convención sobre los Derechos del Niño de 1989; el Comité de Cumplimiento, establecido por la Convención sobre el Acceso a la Información, la Participación del Público en la Toma de Decisiones y el Acceso a la Justicia en Asuntos Ambientales (Convención de Aarhus) de 1998; el Comité sobre los Derechos de las Personas con Discapacidad, creado por la Convención sobre los Derechos de las Personas con Discapacidad de 2006; y el Comité de Derechos Económicos, Sociales y Culturales en virtud del Protocolo Facultativo del Pacto Internacional de Derechos Económicos, Sociales y Culturales de $2008^{74}$.

\footnotetext{
74 CDI, Proyecto de conclusiones 2018, cit., nota 5, pp. 115-116.
} 
Estos órganos se suelen pronunciar a través de observaciones, resoluciones, recomendaciones, comentarios, medidas - entre otros términos utilizados-, los mismos que pueden expresar o dar lugar a un acuerdo ulterior o a una práctica ulterior. Así sucedió, por ejemplo, cuando la Asamblea General de la ONU aprobó el 17 de diciembre de 2015 la Resolución 70/169 por la cual adoptó la definición del derecho humano al agua potable en los mismos términos que lo hizo el Comité de Derechos Económicos, Sociales y Culturales en su Observación General núm. 15 de $2002^{75}$.

Otro caso que puede citarse es el de las conclusiones y observaciones generales formuladas por el Comité de Derechos Humanos para interpretar el Pacto Internacional de Derechos Civiles y Políticos ${ }^{76}$, cuya importancia ha sido resaltada por la Corte Internacional de Justicia en el asunto Ahmadou Sadio Diallo:

«Desde que fue creado, el Comité de Derechos Humanos ha construido un considerable cuerpo de jurisprudencia interpretativa, en particular mediante sus conclusiones en respuesta a las comunicaciones individuales que pueden presentarse ante él con respecto a los Estados parte en el primer Protocolo facultativo, así como mediante sus "observaciones generales".

La Corte observa que, aun cuando en el ejercicio de sus funciones judiciales no está en modo alguno obligada a ajustar su propia interpretación del Pacto a la del Comité, estima que debe dar mucho peso a la interpretación adoptada por este órgano independiente que fue establecido específicamente para vigilar la aplicación de dicho tratado» ${ }^{77}$.

\subsection{Los órganos de control}

Vinculado al punto anterior, refiere Manuel Díez de Velasco que la Corte Internacional de Justicia también ha resaltado el valor de las opiniones de los órganos de control jurisdiccional creados por ciertos tratados de derechos humanos para interpretar las disposiciones contenidas en estos ${ }^{78}$.

Este es el caso, por ejemplo, del Tribunal Europeo de Derechos Humanos, del Tribunal Africano de Derechos Humanos y de los Pueblos, o el de la Corte Interamericana de Derechos Humanos, como órganos jurisdiccionales de control, cuyas interpretaciones deben ser tomadas en cuenta por los Estados parte.

75 Ibid., p. 120.

76 Pacto Internacional de Derechos Civiles y Políticos, adoptado y abierto a la firma, ratificación y adhesión por la Asamblea General en su resolución 2200 A (XXI), de 16 de diciembre de 1966. Véase https://www.ohchr.org/SP/Professionalinterest/Pages/CCPR.aspx.

77 Ahmadou Sadio Diallo (République de Guinée c. République démocratique du Congo), arrêt, CIJ Recueil, 2010, pp. 639 y ss.

78 Conséquences juridiques de l'édification d'un mur dans le territoire palestinien occupé, avis consultatif, CIJ Recueil, 2004, párrs. 97, 109-112. Véase DíEz DE Velasco, M., Instituciones de Derecho Internacional Público, Madrid, Tecnos, 2009, p. 209; y AMERASHinge, C. F., «Interpretation of texts in open International Organizations», British Yearbook of International Law, 1994, núm. 65, pp. 175-209, esp. p. 175. 
Incluso, en el caso particular de la Corte Interamericana de Derechos Humanos, desde el año 2006, a partir del caso Almonacid y otros v. Chile, en no menos de cuarenta y cinco sentencias, este tribunal ha venido señalando el deber de las autoridades internas de los Estados de llevar adelante el denominado control de convencionalidad ${ }^{79}$. En síntesis, esto implica que toda autoridad pública de un Estado, sea esta administrativa o judicial, debe ejercer de oficio un control de convencionalidad, entre la norma jurídica interna, de una parte, y la Convención Americana y demás instrumentos interamericanos de los que el Estado es parte, así como la interpretación que de los mismos ha hecho la Corte, sea por medio de su función contenciosa o consultiva, por otra; debiendo, en caso de encontrar una incompatibilidad, suprimir las normas internas opuestas o expedir aquellas normas conducentes a la efectiva aplicación de la norma o jurisprudencia interamericana ${ }^{80}$.

De esta manera, la interpretación efectuada por la Corte Interamericana respecto de la Convención Americana sobre Derechos Humanos y otros instrumentos ha adquirido carácter vinculante, independientemente de si esta interpretación está plasmada en una sentencia o en una opinión consultiva de este tribunal, la misma que puede estar asentada en un acuerdo o práctica ulterior de las partes.

\subsection{Alcances modificatorios de esta regla}

De otro lado, un importante sector de la doctrina sostiene que las partes de un tratado a través de esta regla de interpretación pueden no solo buscar establecer su verdadero significado, sentido y alcance, sino también pueden darle una significación especial a sus términos, e incluso enmendarlo, ampliarlo o reducirlo en sus alcances y efectos ${ }^{81}$.

Esta posición liberal sobre la posibilidad de modificar tratados vía la aplicación de esta regla ha sido también asumida por la Corte Internacional de

79 Caso Almonacid Arellano y otros v. Chile, Excepciones Preliminares, Fondo, Reparaciones y Costas. Sentencia de 26 de septiembre de 2006, Serie C, núm. 154, párrs. 123-124.

${ }^{80}$ Véase esta evolución en los siguientes casos de la Corte Interamericana de Derechos Humanos: caso Trabajadores Cesados del Congreso (Aguado Alfaro y otros) v. Perú, Excepciones Preliminares, Fondo, Reparaciones y Costas, Sentencia de 24 de noviembre de 2006, Serie C, núm. 158, párr. 128; caso Radilla Pacheco v. México, Excepciones Preliminares, Fondo, Reparaciones y Costas, Sentencia de 23 de noviembre de 2009, Serie C, núm. 209, párrs. 338 y 340; caso Vélez. Loorv. Panamá, Excepciones Preliminares, Fondo, Reparaciones y Costas, Sentencia de 23 de noviembre de 2010, Serie C, núm. 218, párr. 287; caso Atala Riffo y niñas v. Chile, Fondo, Reparaciones y Costas, Sentencia de 24 de febrero de 2012, Serie C, núm. 239, párr. 284; caso Norín Catrimán y otros (Dirigentes, miembros y activistas del Pueblo Indígena Mapuche) v. Chile, Fondo, Reparaciones y Costas, Sentencia de 29 de mayo de 2014, Serie C, núm. 279, párrs. 436, 461 y 464; y en el caso Andrade Salmón v. Bolivia, Fondo, Reparaciones y Costas, Sentencia de 1 de diciembre de 2016, Serie C. núm. 330, párr. 93. NovaK, F., «The System of Reparations in the Jurisprudence of the Inter-American Court of Human Rights», Recueil des Courses de l'Académie de Droit International, vol. 392, 2018, pp. 180-181.

81 Villiger, M., Commentary..., op. cit., nota 23, p. 429 ; Distefano, G., «La pratique subséquente des États parties à un traité», Annuaire français de droit international, vol. 40, 1994, pp. 41-71, esp. pp. 62 y ss; SHaw, M., International Law, Cambridge, Cambridge University Press, 2014, p. 677. 
Justicia, mas no por el Órgano de Apelación de la OMC, que guarda un criterio más conservador ${ }^{82}$.

Como antecedente de esta postura, es pertinente recordar que en el art. 38 del proyecto original de la Convención de Viena sobre Derecho de los Tratados de 1969 se contempló expresamente tal posibilidad, al señalarse: «Todo tratado podrá ser modificado por la práctica ulterior en la aplicación del tratado cuando tal práctica denote el acuerdo de las partes en modificar las disposiciones del tratado» ${ }^{83}$.

No obstante lo antes señalado, la conclusión 7 (3) del Proyecto de conclusiones de la CDI de 2018 asume una posición contraria, al disponer:

«Se presume que las partes en un tratado, mediante un acuerdo o una práctica en relación con la aplicación del tratado, tienen la intención de interpretar el tratado, y no de enmendarlo o modificarlo. La posibilidad de enmendar o modificar un tratado mediante la práctica ulterior de las partes no ha sido reconocida de manera general. El presente proyecto de conclusión se entiende sin perjuicio de las normas relativas a la enmienda o la modificación de los tratados de la Convención de Viena de 1969 y del Derecho internacional consuetudinario».

En efecto, si bien la CDI reconoce que, vía esta regla de interpretación, las partes pueden llevar adelante una interpretación amplia o restringida del contenido de un tratado, no es posible enmendarlo o modificarlo. En primer lugar, sostienen, porque la enmienda o modificación es un procedimiento que tiene sus propias reglas y que está contenido en el art. 39 de la Convención de Viena de 1969; en segundo lugar, porque en el art. 31, párr. 3, del mismo cuerpo normativo no se contempla ni expresa ni implícitamente tal posibilidad, y, en tercer lugar, porque tal supuesto no está considerado en el Derecho internacional general ${ }^{84}$.

Se cita además, en apoyo de esta posición, el informe del Órgano de Apelación de la OMC, dictado en el caso CE-Banano III, donde se afirmó:

«El término "aplicación" que figura en el apartado a) del párrafo 3 del artículo 31 de la Convención de Viena atañe a los casos en que en un acuerdo se especifica la manera en que las normas u obligaciones en vigor tienen que "aplicarse"; el término no connota la creación de nuevas obligaciones o la prórroga de obligaciones existentes» ${ }^{85}$.

En el mismo sentido se ha pronunciado la Corte Internacional de Justicia en el Asunto Fábricas de celulosa en el río Uruguay. Incluso se afirma por parte de la CDI que desde que se aprobó la Convención de Viena de 1969, los

\footnotetext{
82 Sinclair, I., The Vienna Convention on the Law of Treaties, Manchester, Manchester University Press, 1984, p. 137; Nolte, G., op. cit, nota 23, p. 141.

83 CDI, Anuario, 1966, cit., nota 21, p. 200.

84 CDI, Proyecto de conclusiones 2018, cit., nota 5, p. 62.

85 OMC, Informe del Órgano de Apelación, Comunidades Europeas-Régimen de la importación, venta y distribución de bananos, 22 de diciembre de 2008, párrs. 391-393, citado en CDI, Proyecto de conclusiones 2018, cit., nota 5, p. 62.
} 
tribunales internacionales se han abstenido, en su mayoría, de reconocer esta posibilidad de modificación ${ }^{86}$.

Esta última afirmación y la postura en general de la CDI respecto a la improcedencia de modificar un tratado vía la aplicación de esta regla de interpretación resulta en nuestro concepto bastante discutible, dado el amplio apoyo doctrinario y jurisprudencial que existe a favor de permitir la enmienda de tratados vía la conducta ulterior.

La propia Corte Internacional de Justicia ha dejado abierta esta posibilidad, como la propia CDI lo reconoce ${ }^{87}$. En efecto, en el Asunto del límite terrestre y marítimo entre Camerún y Nigeria este tribunal aceptó que la modificación de un tratado vía práctica ulterior «no puede ser excluida totalmente desde el punto de vista jurídico» ${ }^{88}$. Asimismo, coincidimos con Marcelo Kohen cuando sostiene que los pocos casos arbitrales donde no se ha permitido la modificación vía interpretación han sido la excepción ${ }^{89}$. En el mismo sentido, el Tribunal Europeo de Derechos Humanos ha consagrado que la práctica ulterior puede modificar el tratado que interpreta. Así, en el caso Öcalan c. Turquía, este tribunal afirmó:

«que una práctica establecida en el seno de los Estados miembros podía dar lugar a una modificación del Convenio. En ese asunto, el Tribunal aceptó que una práctica ulterior en materia de política penal nacional, en forma de una abolición generalizada de la pena de muerte, podría entenderse como establecimiento del acuerdo de los Estados contratantes en derogar la excepción prevista en el artículo 2, párrafo 1, y, de ese modo, eliminar un límite textual al margen de la interpretación evolutiva del artículo $3 »^{90}$.

Y es que no se comprende por qué la voluntad de las partes en un tratado, libre e indubitablemente expresada con posterioridad a su celebración a través de un acto o práctica ulterior, no pueda implicar la modificación de dicho instrumento. Si bien la Convención de Viena establece un procedimiento para la modificación o enmienda del tratado, ello no niega que puedan existir otras formas libres y voluntarias de modificación del texto.

Esto por supuesto no puede llevar a extremos, es decir, la modificación vía interpretación deberá ser solo aceptada, siempre que la voluntad de todas las partes intervinientes haya sido manifestada de manera clara e indubitable, en particular, en los tratados que afecten intereses vitales de los Estados parte.

86 Usines de pâte à papier sur le fleuve Uruguay (Argentine c. Uruguay), mesures conservatoires, CIJ Recueil, 2006, pp. 63 y 66, CDI, Proyecto de conclusiones 2018, cit., nota 5, p. 64.

87 CDI, Proyecto de conclusiones 2018, cit., nota 5, p. 66.

88 Frontière terrestre et maritime entre le Cameroun et le Nigéria (Cameroun c. Nigéria; Guinée Équatoriale (intervenant)), arrêt, CIJ Recueil, 2002, pp. 303 y ss.

89 KoHen, M., «Keeping subsequent agreements and practice in their right limits», en Nolte, G. (ed.), Treaties and Subsequent Practice, Oxford, Oxford University Press, 2013, pp. 34-45, esp. pp. 34 y ss.

90 Öcalan c. Turkey, núm. 46221/99, párr. 163, TEDH 2005, CDI, Proyecto de conclusiones 2018, cit., nota 5 , p. 66. 


\subsection{La conducta ulterior y la interpretación evolutiva}

La conducta ulterior puede ser una regla relevante para contribuir a determinar si la intención presunta de las partes al celebrar un tratado era atribuir, a un término allí utilizado, un sentido susceptible de evolucionar en el tiempo o por el contrario, estar sujeto al Derecho vigente al momento de su celebración ${ }^{91}$. Esto lo ha reconocido la CDI en su conclusión $8^{92}$, al igual que la jurisprudencia internacional.

Así, en el Asunto de la Disputa sobre Derechos de Navegación y Derechos Conexos, entre Costa Rica y Nicaragua ${ }^{93}$, se determinó cómo la conducta ulterior puede contribuir en la determinación de si a un término se le debe atribuir, o no, un sentido susceptible de evolucionar en el tiempo. Concretamente, se interpretó el término "comercio», empleado en el tratado de 1858 celebrado por ambos países. Se indicó que, al ser un término genérico, las partes necesariamente habían estado al tanto de que su significado era probable de evolucionar en el tiempo ${ }^{94}$. Además, la Corte señaló que el tratado había estado en vigor por un largo periodo y concluyó que se presumía que las partes habían tenido la intención de que el término "comercio» tuviera un significado susceptible de evolucionar ${ }^{95}$.

No obstante, en otros casos, como Mangouras c. España, es posible identificar que, ante la posibilidad de una interpretación evolutiva, el tribunal optó por un resultado distinto, en razón de la práctica ulterior de las partes ${ }^{96}$.

\subsection{Criterios de valoración de la regla}

Finalmente, con relación a los criterios a tener en cuenta para valorar el acuerdo y la práctica ulterior como medios valederos de interpretación de los tratados, la CDI, en la conclusión 9 del Proyecto de 2018 y en sus comentarios, plantea algunos parámetros.

En este sentido, sostiene que se debe tener en cuenta la claridad del acuerdo o de la práctica ulterior (esto es, que sea cierta, precisa, evidente); la especificidad de ambos (es decir, que el acuerdo o práctica sea determinada,

91 BJORge, E., «The Vienna Rules, Evolutionary Interpretation, and the Intentions of the Parties», en Bianchi, A., Peat, D. y Windsor, M. (eds.), Interpretation in International Law, Oxford, Oxford University Press, 2015, pp. 189-204, p. 203.

92 CDI, Proyecto de conclusiones 2018, cit., nota 5, p. 64.

93 Différend relatif à des droits de navigation et des droits connexes (Costa Rica c. Nicaragua), arrêt, CIJ Recueil, 2009, p. 242. Véase Dawidowicz, M., «The Effect of the Passage of Time on the Interpretation of Treaties: Some Reflections on Costa Rica v. Nicaragua», Leiden Journal of International Law, vol. 24, 2011, núm. 1, pp. 201-222, pp. 207 y ss.

94 Ibid., p. 243.

95 Id.

96 BJorge, E., The Evolutionary Interpretation of Treaties, Oxford, Oxford University Press, 2014, pp. $78-79$. 
concreta, definida); el momento en que se desarrolló la práctica (citándose como ejemplo la controversia marítima Perú c. Chile resuelta por la Corte Internacional de Justicia, donde se tomó en cuenta la práctica contemporánea al acuerdo tácito que definió parte de la frontera) ${ }^{97}$; la importancia dada por las partes al acuerdo o a la práctica (esto es, que sea comprendida por ambas como una verdadera interpretación del acuerdo y no como un mero comentario sin relevancia o significación jurídica); la repetición de la práctica (lo que alude a la frecuencia o constancia de la misma); la forma de la práctica (de qué manera fue efectuada); la coherencia de la práctica (ello se refiere a la uniformidad), y la pertinencia (respecto de la norma que se pretende interpretar), entre otros ${ }^{98}$.

Si bien este listado de criterios a tener en cuenta cuando estemos frente a un acuerdo o práctica ulterior no es cerrado, en tanto pueden sumarse otros, sirve como una guía a tener en cuenta para una correcta aplicación de este principio de interpretación de los tratados.

\section{REFLEXIÓN FINAL}

Lo expuesto en este estudio nos permite concluir que la doctrina, pero sobre todo la jurisprudencia internacional y la CDI en su reciente Proyecto de conclusiones de 2018, han permitido un amplio desarrollo respecto del contenido y alcances de la conducta ulterior de las partes como regla general y principal de interpretación de los tratados. Si bien ello no implica que la regla carezca de ámbitos aún por precisar o alcanzar consensos, actualmente podemos afirmar que se trata de una de las reglas de interpretación de los tratados con mayor grado de caracterización.

En todo caso, será particularmente importante observar el nivel de aceptación o no que alcance el proyecto de la CDI antes referido, tanto por parte de la jurisprudencia internacional como por la práctica interestatal, pues ello permitirá que esta regla alcance un mayor nivel de desarrollo y precisión.

\section{RESUMEN}

\section{LA CONDUCTA ULTERIOR DE LAS PARTES COMO REGLA PRINCIPAL DE INTERPRETACIÓN DE LOS TRATADOS}

El presente estudio analiza los recientes avances y desarrollos en torno a una de las reglas más importantes de interpretación de los tratados: la conducta ulterior de las partes. En este sentido, este estudio tiene como propósito establecer el contenido y los alcances de este principio principal de interpretación, recogido tanto por la Convención de Viena de 1969 sobre Derecho de los tratados como por la costumbre internacional, teniendo en cuenta su aplicación por parte de la jurisprudencia internacional, pero también el Proyecto de conclusiones sobre los acuerdos ulteriores y la práctica ulterior en relación con la inter-

97 Différend maritime (Pérou c. Chili), arrêt, CIJ Recueil, 2014, pp. 3 y 50.

98 CDI, Proyecto de conclusiones 2018, cit., nota 5, pp. 76-80. 
pretación de los tratados aprobado por la CDI, en agosto de 2018. Esto será especialmente útil para los aplicadores del Derecho internacional, tanto en el interior de los Estados como por las entidades de carácter internacional, en su permanente labor de interpretación, aplicación e implementación de los tratados internacionales.

Palabras clave: acuerdo ulterior, práctica ulterior, prueba objetiva, CDI, interpretación de tratados.

\section{ABSTRACT \\ SUBSEQUENT CONDUCT OF THE PARTIES AS MAIN RULE FOR TREATY INTERPRETATION}

This study analyzes recent advances and developments around one of the most important rules of treaty interpretation: the subsequent conduct of the parties. In this sense, this study aims to establish the content and scope of this main principle of interpretation, reflected both by the 1969 Vienna Convention on the Law of Treaties and by international custom, taking into account its application by international jurisprudence but also the draft conclusions on subsequent agreements and subsequent practice in relation to the interpretation of treaties adopted by the International Law Commission in August 2018. This will be especially useful for those who apply international law, both within the States as well as at international entities, in their permanent work of construing, applying and implementing international treaties.

Keywords: subsequent agreement, subsequent practice, objective evidence, ILC. 